\title{
THE STEADY-STATE IMPEDANCE OPERATOR OF A LINEAR PERIODICALLY TIME-VARYING ONE-PORT NETWORK AND ITS DETERMINATION
}

\author{
RADOSŁAW KŁOSIŃSKI \\ Institute of Electrical Metrology \\ University of Zielona Góra, ul. Podgórna 50, 65-246 Zielona Góra, Poland \\ e-mail: R.Klosinski@ime.uz.zgora.pl
}

\begin{abstract}
The main subject of the paper is the description and determination of the impedance operator of a linear periodically timevarying (LPTV) one-port network in the steady-state. If the one-port network parameters and the supply vary periodically with the same period, the network reaches a periodic steady state. However, the sinusoidal supply may induce a nonsinusoidal voltage or current. It is impossible to describe such a phenomenon by means of one complex number. A periodically time-varying one-port network working in a steady-state regime can be described with a circular parametric operator. Within the domain of discrete time, such an operator takes the form of a matrix with real-valued entries. The circular parametric operator can be transformed into the frequency domain using a two-dimensional DFT. This description makes it possible to quantitatively assess LPTV system input and output harmonics aliasing. The paper also presents the derivation and the proof of convergence of an iteration scheme for the identification of circular parametric operators. The scheme may be used to determine the impedance of an LPTV one-port network. Some results of computer simulations are shown.
\end{abstract}

Keywords: periodically time-varying impedance, periodic steady state, linear periodically time-varying system, circular parametric operator, harmonics aliasing, identification.

\section{Introduction}

The knowledge of the parameters of an electrical distribution power system is necessary for effective control of electronic compensators, for the localization of harmonic distortion sources, and to observe system behavior (Mikołajuk and Staroszczyk, 2004). Time-varying circuits, e.g., switching converters or thyristor-driven loads, and non-linear system components, e.g., transformers, saturated chocking coils or diode-equipped loads, change their parameters synchronously with the power voltage and cause deformations in the current. These one-port networks can be treated as single input, single output linear periodically time-varying systems, as depicted in Fig. 1

The subject of the paper is the determination of steady-state behavior of linear periodically time-varying (LPTV) one-port networks and, in general, of single input, single output LPTV systems (SISO LPTV). The latter are considered as a bridge between the well-studied linear time invariant (LTI) model and real nonlinear timevarying systems stimulated periodically. The LPTV model is linear, i.e., it meets the superposition principle, and it can generate and shift harmonic components like

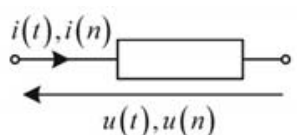

(a)

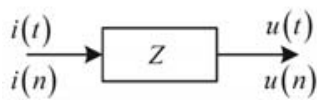

(c)

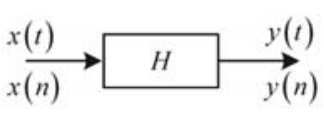

(b)

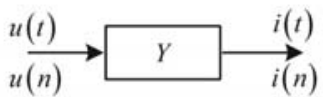

(d)
Fig. 1. One-port network treated as an SISO system: (a) oneport network, (b) transmittance, (c) impedance, (d) admittance.

nonlinear systems. Parameters of an LPTV system change periodically. To obtain the steady state, a common period of LPTV system parameter changes and stimulation signal has to exist. The steady-state current (or voltage) of an LTI one-port network excited by a sinusoidal voltage (or current) is also sinusoidal with the same frequency. The relationship between its terminal signals phasors is described by an impedance (or admittance) phasor, which 
is a complex number. The situation is different in the case of LPTV one-port networks, which can also reach a periodic steady state, but a sinusoidal supply may give rise to a non-sinusoidal voltage or current. The mathematical description of such phenomena by means of one complex number is impossible. The problem consists in the description of an LPTV one-port network in the steady state, which is similar to the description of an LTI one-port network using an impedance phasor.

There are many publications related to LPTV systems used in control systems, multi-rate signal processing and telecommunications. Some common representations of LPTV systems including linear time invariant models obtained by blocking, linear switched timevarying (LSTV) systems and alias-component matrices are described in (Mehr and Chen, 2002) and the references therein. The description of the LPTV system as a time invariant block system is presented in (Meyer and Burrus, 1975). The so-called system function of a variable network and a bi-frequency system function are used in (Zadeh, 1950) for frequency analysis of variable networks. A state space model with time-varying coefficients is usually in use to describe linear time-varying (LTV) systems universally, e.g., (Kaczorek, 2001; Bru et al., 2004). A discrete time domain state space model, transition matrices and pseudo-modal parameters are used to describe and identify LTV systems in (Liu, 1997; 1999; Liu and Deng, 2005). Subspace model identification algorithms that allow the identification of an LTV state space model from a set of input-output measurements are presented in (Verhagen and Yu, 1995).

A complete and clear picture on time-invariant representations for discrete-time periodic systems is described in (Bittanti and Colaneri, 1999; 2000), where the concepts of a periodic transfer function and generalized frequency response are used. The following four reformulations are discused: time lifted, cyclic, frequency lifted and Fourier. They are obtained from input-output and state-space representations. The authors emphasize that the reformulation of a periodic system always leads to a particular class of time invariant models. The characteristics of these models (structure, dimensions, etc.) cannot be neglected, otherwise entering a mess of nonfeasibility/noncausality issues. The paper (Hu et al., 2007) presents a new kind of linear model with partially variant coefficients and iterative identification algorithms. In (Mikołajuk and Staroszczyk, 2003; 2004; Staroszczyk, 2002; Staroszczyk and Mikołajuk, 2004), some methods of power system impedance identification as an LPTV model are presented. The LPTV model used requires 2D time-frequency system description. The time-varying ARMA (autoregressive moving average) model is also used. The power system is tested with short (1-3 ms) current impulse excitations. Impedance frequency characteristics are determined by means of a method called the empirical transfer function estimate (EFTE) based on the input/output transformed (using DFT) signals identification experiment. Discrete time LPTV systems can also be modeled by discrete time wavelets. It makes the system identification robust to narrow-band or impulse noise (Doroslovacki et al., 1998). The papers (Liu et al., 2003; Tam et al., 2006) present an approach to finding steady-state waveforms based on wavelet approximation of power electronics circuits that are described by means of a time-varying state-space equation.

In this paper, the periodic steady state of an LPTV one-port network is taken into consideration. To obtain a periodic steady state, one-port network parameter changes and the power supply should have the same period, which is the least common multiple of the one-port network period and the supply period. It is assumed that the period of stimulation signal changes is an integer multiplicity of the system parameter changes period. This assumption imposes some restrictions on the application space. However, it returns significant simplification and convenience. In this situation, the period of system response is equal to the stimulation period. The relationship between steadystate voltage and current is described by means of the socalled circular parametric operator (CPO). It is a circular form of the description based on the time-varying pulse response. To describe an SISO LPTV system in this way, no restriction on system parameter changes is needed (no restriction was found in references concerned with LPTV systems).

For signal processing, it is better to use the discrete time domain, where the CPO describes the relationship between vectors of samples of one period of input and output signals and takes the form of a matrix with real-valued entries. It is a circular form of the description known as periodic Markov coefficients linked to the pulse response of the system (see, e.g., (Bittanti and Colaneri, 2000)), and it is a generalization of circular convolution used for LTI systems. The description of an LPTV system by means of a CPO was introduced for the first time by Siwczyński to implement a new numerical operator method for nonlinear systems analysis described in (Siwczyński, 1987). Later, CPOs were used in algorithms of optimal operational condition determination of real voltage sources with periodically time-varying or non-linear inner impedance and load one-port networks (Siwczyński et al., 1993; Siwczyński, 1995; Siwczyński and Kłosiński, 1997a).

Discrete time domain description of an LPTV system and signals implies a restriction on signals and parameter changes. Exact discrete representation of signals and system is possible if input signal and parameter changes spectra are bounded. Then, as a result of modulation, the limit of the output spectrum is equal to the sum of input and parameter changes spectrum limits. The sampling frequency should be at least two times greater than this limit. If the spectrum of the system parameter changing 
is not bounded, it is impossible to obtain an accurate discrete model, but it is feasible to select proper sampling frequency to obtain acceptable accuracy of the discrete model of the LPTV system.

The relation between input and output harmonics complex phasors of the LPTV system can be obtained by means of a two dimensional discrete Fourier transformation. This topic is widely discussed in Section 3.

The impedance or admittance CPO of a one-port network can be determined on the basis of measurements. To obtain the fully identified circular parametric $N \times N$ matrix, a set of $N$ pairs of system stimulus and response signals is needed. The stimulus signals have to be linearly independent. Sometimes, it is difficult to obtain such a complete set of measured signals. In these cases, an identification iteration scheme which is presented in this paper may be useful. The proposed method is based on the determination of the CPO describing the system with the smoothest parameter changes. An attempt to apply the iteration scheme to optimal control of a compensation circuit in a simple power system, described in (Kłosiński, 2005), can be a good example of its effectiveness. The combination of the identification scheme and the optimal current determination algorithm allows optimal compensation in a linear time-varying circuit without previous knowledge of circular parametric operators of the source and load impedance. Their partial on-line identification is sufficient.

If a nonlinear system can achieve a periodic steady state, its parameters change periodically, coherently with the input. In this situation, the behavior of the non-linear system can be described by means of a set of CPOs. For example, CPOs were used to describe a nonlinear current transformer in (Kłosiński and Kozioł, 2007). From this description, it is possible to estimate the current flowing through the primary of the transformer on the basis of secondary current samples. CPOs describing a real CT were calculated using an identification scheme based on measurements. The obtained results show that the usage of CPOs gave good results.

The CPO identification method applied resembles the algorithm called the smooth periodic escape (SPE), used to design time-varying compensator networks (Siwczyński, 1995; Siwczyński and Kłosiński, 1997a; 1997b). The derivation and properties of the identification scheme were presented in (Kłosiński, 2005; 2006; 2007). The identification scheme is iterative, and it was not easy to prove its convergence because of its special construction. In this paper, for the first time the proof of iterative identification scheme convergence to a unique solution is presented. Apart from theoretical deliberations, the paper also presents some results of computer simulations.

In the paper, linear causal single input single output systems are considered. It is assumed that all signals are noise-free, sampled coherently with adequately high sampling frequency.

\section{Circular parametric operator}

The relation of the input signal $x(t)$ to the output signal $y(t)$ for an SISO LTV system (Fig. 11b)) can be described with a differential equation of variable coefficients:

$$
\sum_{i=0}^{q} a_{i}(t) y^{(i)}(t)=\sum_{i=0}^{r} b_{i}(t) x^{(i)}(t) .
$$

Equation (1) can be solved with the integral operator $H$ :

$$
y(t)=H x(t)=\int_{-\infty}^{\infty} h(t, \tau) x(\tau) \mathrm{d} \tau .
$$

The operator kernel $h(t, \tau)$ is the response of the system to Dirac's impulse $\delta(t)$. For a time-varying system, it is a function of two variables - it depends on the current time $t$ and on the time instant $\tau$ when an impulse is applied to the system.

The discrete time description is more useful for practical applications. Within the discrete time domain, the relation of the input signal $x(n)$ to the output signal $y(n)$ for an LTV system can be described by means of a difference equation of variable coefficients,

$$
\sum_{i=0}^{q} A_{i}(n) y(n-i)=\sum_{i=0}^{r} B_{i}(n) x(n-i) .
$$

Equation (3) can be solved with the operator set in the form of the following sum:

$$
y(n)=H x(n)=\sum_{m=-\infty}^{\infty} h(n, m) x(m) .
$$

The operator kernel $h(n, m)$ is the response to Kronecker's impulse applied at the moment $m$. In the case of a time-varying system, it is a function of two variables, i.e., it depends on the current discrete time $n$, and on the instant $m$ in which the impulse has been applied. For the $N$-periodic input signal, $x(n+N)=x(n)$, the response can be determined using the following formula (Siwczyński, 1995):

$$
y(n)=\tilde{H} x(n)=\sum_{m=0}^{N-1} \tilde{h}(n, m) x(m),
$$

where the circular impulse response is defined as

$$
\tilde{h}(n, m)=\sum_{p=-\infty}^{\infty} h(n, m-p N) .
$$

For a linear time-varying system whose parameters vary with period $N$, the impulse response satisfies the property

$$
h(n+N, m+N)=h(n, m)
$$


and, therefore,

$$
\begin{aligned}
\tilde{h}(n+N, m) & =\sum_{p=-\infty}^{\infty} h(n+N, m-p N) \\
& =\sum_{p=-\infty}^{\infty} h(n, m-(p+1) N)=\tilde{h}(n, m) .
\end{aligned}
$$

This means that the response $y(n)$ to an $N$-periodic stimulus signal $x(n)$ is periodic as well:

$$
\begin{aligned}
y(n+N) & =\sum_{m=0}^{N-1} \tilde{h}(n+N, m) x(m) \\
& =\sum_{m=0}^{N-1} \tilde{h}(n, m) x(m)=y(n) .
\end{aligned}
$$

The response of an LPTV system of period $N$ to an $N$-periodic stimulus can be determined by means of the so-called circular parametric operator:

$y(n)=\tilde{H} x(n)=\sum_{m=0}^{N-1} \tilde{h}(n, m) x(m), \quad n=0,1, \ldots, N-1$.

The circular parametric operator can be given in the form of the following matrix (Siwczyński, 1995):

$$
\boldsymbol{y}=\tilde{\boldsymbol{H}} \boldsymbol{x},
$$

where $\boldsymbol{x}=[x(0) x(1) \cdots x(N-1)]^{T}$ and $\boldsymbol{y}=$ $[y(0) y(1) \cdots y(N-1)]^{T}$ are vectors of samples of one period of the stimulus and response signals, respectively, and

$$
\tilde{\boldsymbol{H}}=\left[\begin{array}{cccc}
\tilde{h}_{0,0} & \tilde{h}_{0,1} & \cdots & \tilde{h}_{0, N-1} \\
\tilde{h}_{1,0} & \tilde{h}_{1,1} & \cdots & \tilde{h}_{1, N-1} \\
\cdots & \cdots & & \cdots \\
\tilde{h}_{N-1,0} & \tilde{h}_{N-1,1} & \cdots & \tilde{h}_{N-1, N-1}
\end{array}\right]
$$

is the circular parametric operator (circular parametric matrix), which is an $N \times N$ matrix with real-valued entries, $\tilde{h}_{n, m}=\tilde{h}(n, m)$.

For an LTI system, the impulse response is a function of one variable:

$$
h(n, m) \rightarrow h(n-m) .
$$

Then, the circular parametric matrix introduced in the equation (12) passes to the form of a circular (Toeplitz) matrix:

$$
\tilde{\boldsymbol{H}}_{L T I}=\left[\begin{array}{cccc}
\tilde{h}_{0} & \tilde{h}_{N-1} & \cdots & \tilde{h}_{1} \\
\tilde{h}_{1} & \tilde{h}_{0} & \cdots & \tilde{h}_{2} \\
\cdots & \cdots & & \cdots \\
\tilde{h}_{N-1} & \tilde{h}_{N-2} & \cdots & \tilde{h}_{0}
\end{array}\right] .
$$

\section{Frequency domain representation of the CPO}

The $N$-periodic input signal can be expressed in terms of the Fourier series of harmonics phasors $X_{m}$ :

$$
x(n)=\frac{1}{2} \sum_{m=0}^{N-1} X_{m} w^{n m}, \quad n=0,1, \ldots, N-1,
$$

where $w=\exp (j 2 \pi / N)$. The values of complex phasors can be calculated from the samples of $x(n)$ using the relation

$$
X_{m}=\frac{2}{N} \sum_{n=0}^{N-1} x(n) w^{-n m} .
$$

The expressions (15) and (16) can be described in matrix form

$$
\begin{aligned}
\boldsymbol{x} & =\frac{1}{2} \boldsymbol{F} \overline{\boldsymbol{X}}, \\
\overline{\boldsymbol{X}} & =2 \boldsymbol{F}^{-1} \boldsymbol{x},
\end{aligned}
$$

where $\boldsymbol{x}$ is a vector of samples of one period of the signal, $\overline{\boldsymbol{X}}$ stands for a vector containing the harmonics phasors $X_{m}$ and the adjoint harmonics phasors, and $\boldsymbol{F}$ is a Fourier matrix defined as follows $(w=\exp (2 \pi / N)$ :

$$
[\boldsymbol{F}]_{n, m}=w^{n m}, \quad n, m=0,1, \ldots, N-1 .
$$

This matrix has the property

$$
\boldsymbol{F}^{-1}=\frac{1}{N} \boldsymbol{F}^{*},
$$

where $\boldsymbol{F}^{*}$ is the conjugate transpose of $\boldsymbol{F}$.

To determine the frequency domain version of the CPO, one should multiply both sides of 11 by $2 \boldsymbol{F}^{-1}$ and substitute (17). Taking into account (18) with reference to the signal $\boldsymbol{y}$, we obtain

$$
\overline{\boldsymbol{Y}}=\boldsymbol{F}^{-1} \tilde{\boldsymbol{H}} \boldsymbol{F} \overline{\boldsymbol{X}} .
$$

Then, the spectral circular parametric operator (SCPO) takes the following form (Siwczyński, 1995):

$$
\overline{\tilde{\boldsymbol{H}}}=\boldsymbol{F}^{-1} \tilde{\boldsymbol{H}} \boldsymbol{F} .
$$

The SCPO $\overline{\tilde{\boldsymbol{H}}}\left(k_{y}, k_{x}\right)$ (where $k_{x}$ and $k_{y}$ are the input and output harmonic enumeration numbers, respectively) offers a possibility for quantitative assessment of input and output harmonics aliasing. This matrix is a special case for the periodic steady state of the so-called alias component matrix described in (Shenoy et al., 1994), the frequency-response matrix for the LPTV system described in (Chen and Qiu, 1997), a discrete version of the so-called bi-frequency system function used in (Zadeh, 1950), and a harmonic transfer function or a frequency lifted transfer function in the case of the periodic steady 
state described in (Bittanti and Colaneri, 1999; 2000). The coefficients of the SCPO matrix lying outside the diagonal determine the manner in which the harmonics of the input signal are mapped into other harmonics at the system output. The $k$-th column of the spectrum operator describes the way in which the $k$-th harmonic of a given stimulus is mapped onto the harmonics of the output signal. The $l$-th line of the spectrum matrix includes entries decisive in the manner of processing all stimulus harmonics into the $l$-th harmonic of the system response. Each harmonic phasor of the response signal is dependent on each stimulus harmonic through two complex coefficients multiplied by the harmonic phasor and the adjoint harmonic phasor, respectively. It is clear that it is impossible to precisely describe an LPTV system in a similar way to an LTI system using a single one-dimensional frequency response.

For an LTI system, the matrix $\tilde{\boldsymbol{H}}_{L T I}$ is circular (14), and, after its transformation according to 22) into the frequency domain, the matrix $\overline{\tilde{\boldsymbol{H}}}_{L T I}\left(k_{y}, k_{x}\right)$ takes the form of a diagonal matrix. In this case, the "separation" of harmonics is clearly visible. Diagonal entries of the matrix are samples of the frequency response of the LTI system.

\section{Determination of the CPO on the basis of measurements}

The identification of a circular parametric operator consists in determining coefficients of the matrix $\tilde{\boldsymbol{H}}$ as a function of a set of $K$ stimuli and $K$ responses of the system. To achieve this, the following relation derived from (11) is used:

$$
\tilde{\boldsymbol{H}} \boldsymbol{X}=\boldsymbol{Y},
$$

where $\boldsymbol{X}$ and $\boldsymbol{Y}$ are matrices of $K$ stimulus and response signals, respectively. Each of these matrices' columns contains samples of one period of one signal. The way of CPO matrix determination depends on the number $K$.

In the case where $K=N$, i.e., the number of stimulus or response signals $K$ equals the size $N$ of the square circular parametric matrix, the problem has an unequivocal solution:

$$
\tilde{\boldsymbol{H}}=\boldsymbol{Y} \boldsymbol{X}^{-1} .
$$

The existence of the solution given in (24) relies on the linear independence of input signals. In other words,

$$
\operatorname{det}(\boldsymbol{X}) \neq 0 \text {. }
$$

In the case where $K>N$, the equation (23) is overdetermined and the matrix $\tilde{\boldsymbol{H}}$ is estimated by means of the least squares method. The distribution of input samples is approximated as a multivariate linear function. The entries of the $n$-th line of the matrix $\tilde{\boldsymbol{H}}$ are treated as sought coefficients of the linear function that results from (23):

$$
\boldsymbol{y}_{n}^{T}=\boldsymbol{h}_{n}^{T} \boldsymbol{X}
$$

where $\boldsymbol{h}_{n}=\left[\begin{array}{llll}h_{n, 0} & h_{n, 1} & \cdots & h_{n, N-1}\end{array}\right]^{T}$ is a vector of entries of the $n$-th line of the matrix $\tilde{\boldsymbol{H}}$, and $\boldsymbol{y}_{n}=$ $\left[\begin{array}{llll}y_{n, 0} & y_{n, 1} & \cdots & y_{n, K-1}\end{array}\right]^{T}$ is a vector containing the $n$-th sample of all $K$ response signals (or, equivalently, the entries of the $n$-th line of the matrix $\boldsymbol{Y}$ ). The least squares solution of the approximation problem for the $n$-th line of the matrix $\tilde{\boldsymbol{H}}$ takes the following form:

$$
\boldsymbol{h}_{n}=\left(\boldsymbol{X} \boldsymbol{X}^{T}\right)^{-1} \boldsymbol{X} \boldsymbol{y}_{n}
$$

The whole matrix $\tilde{\boldsymbol{H}}$ may be estimated as

$$
\tilde{\boldsymbol{H}}^{T}=\left(\boldsymbol{X} \boldsymbol{X}^{T}\right)^{-1} \boldsymbol{X} \boldsymbol{Y}^{T} .
$$

In order to implement the solution (28), the invertibility of the matrix $\left(\boldsymbol{X} \boldsymbol{X}^{T}\right)$ is necessary, and thus the following condition has to be fulfilled:

$$
\operatorname{det}\left(\boldsymbol{X} \boldsymbol{X}^{T}\right) \neq 0 \text {. }
$$

The condition means that the matrix of stimulus signals $\boldsymbol{X}$ has to contain a subset of $N$ linearly independent signals.

In the case where $K<N$, the matrix equation 23. has an infinite number of solutions. An optimal solution should be chosen. One can propose to seek an operator which describes a system with the smoothest parameter changes. This seems to be a proper solution because, if it is possible, an LTI model may be found. It would be a significant simplification of the examined system description. This criterion implies minimization increments of the coefficients of the matrix $\tilde{\boldsymbol{H}}$ in the direction of the main diagonal. Such a criterion choice originates from the fact that, in the case of an LTI system, a relationship between input and output signals in the periodic steady state is described by means of the circular (Toeplitz) matrix (14). In this case, the increments equal zero. On the other hand, the calculated matrix $\tilde{\boldsymbol{H}}$ should fulfill the relation (23). Therefore, a constrained optimization problem may be defined as follows:

$$
\begin{aligned}
\left(\Delta \boldsymbol{h}_{n}\right)^{T} \Delta \boldsymbol{h}_{n} & \rightarrow \min \\
\boldsymbol{X}^{T} \boldsymbol{h}_{n} & =\boldsymbol{y}_{n},
\end{aligned}
$$

(For notation, see (26).) The vector of increments of $\tilde{\boldsymbol{H}}$ elements for the $n$-th line is defined as

$$
\begin{gathered}
\Delta \boldsymbol{h}_{n}=\left[\begin{array}{cccc}
\Delta h_{n, 0} & \Delta h_{n, 1} & \cdots & \Delta h_{n, N-1}
\end{array}\right]^{T}, \\
\Delta h_{n, m}=h_{n, m}-h_{n \ominus 1, m \ominus 1},
\end{gathered}
$$

where $\ominus$ is a subtraction mark of the modulo $N$. The vector $\Delta \boldsymbol{h}_{n}$ can be also defined with the use of a circular unit delay matrix of the form

$$
\boldsymbol{P}_{\mathbf{1}}=\left[\begin{array}{cccc}
0 & \cdots & 0 & 1 \\
1 & \cdots & 0 & 0 \\
\cdots & \cdots & \cdots & \cdots \\
0 & \cdots & 1 & 0
\end{array}\right]
$$


as follows:

$$
\Delta \boldsymbol{h}_{n}=\boldsymbol{h}_{n}-\boldsymbol{P}_{1} \boldsymbol{h}_{n-1} .
$$

The choice of the criterion 30 with the constraint 31), implies the search of the CPO describing a system with a reduced parameter variability realizing (23). Equation (31) results from (23).

The optimization problem (30), (31) can be solved using the Lagrange multipliers method in a specific manner similar to that presented in (Siwczyński, 1995; Siwczyński and Kłosiński, 1997a; 1997b). Lagrange's functional is

$$
f\left(\boldsymbol{h}_{n}, \Lambda\right)=\left(\Delta \boldsymbol{h}_{n}\right)^{T} \Delta \boldsymbol{h}_{n}+\left(\boldsymbol{h}_{n}^{T} \boldsymbol{X}-\boldsymbol{y}_{n}^{T}\right) \Lambda,
$$

where $\Lambda=\left[\begin{array}{llll}\lambda_{0} & \lambda_{1} & \cdots & \lambda_{K-1}\end{array}\right]^{T}$ is a vector of Lagrange multipliers. If the functional is differentiable, it is convenient to search for its extreme by means of the Fréchet differential. The gradient is determined automatically and equating it to zero yields the necessary and sufficient condition of the minimum or maximum of the functional.

The determination and equating the gradient to zero alone yields just a necessary condition for the extreme existence. Taking into consideration that

$$
\begin{aligned}
\delta \Delta \boldsymbol{h}_{n} & =\delta\left(\boldsymbol{h}_{n}-\boldsymbol{P}_{1} \boldsymbol{h}_{n-1}\right) \\
& =\left(\boldsymbol{h}_{n}+\delta \boldsymbol{h}_{n}-\boldsymbol{P}_{1} \boldsymbol{h}_{n-1}\right)-\left(\boldsymbol{h}_{n}-\boldsymbol{P}_{1} \boldsymbol{h}_{n-1}\right) \\
& =\delta \boldsymbol{h}_{n},
\end{aligned}
$$

the Fréchet differential of Lagrange's functional for the vector $\boldsymbol{h}_{n}$ is

$$
\begin{aligned}
\delta f & \left(\boldsymbol{h}_{n}, \Lambda\right) \\
& =f\left(\boldsymbol{h}_{n}+\delta \boldsymbol{h}_{n}, \Lambda\right)-f\left(\boldsymbol{h}_{n}, \Lambda\right) \\
& =\left(\delta \boldsymbol{h}_{n}\right)^{T}\left(2 \Delta \boldsymbol{h}_{n}+\boldsymbol{X} \Lambda\right)+\left(\delta \boldsymbol{h}_{n}\right)^{T}\left(\delta \boldsymbol{h}_{n}\right)
\end{aligned}
$$

where $\delta \boldsymbol{h}_{n}=\left[\begin{array}{llll}\delta h_{n, 0} & \delta h_{n, 1} & \cdots & \delta h_{n, N-1}\end{array}\right]^{T}$. If the minimum of Lagrange's functional (36) is obtained for $\boldsymbol{h}_{n}$, any variation $\Delta \boldsymbol{h}_{n}$ causes an increase in the functional value. The minimum condition is that, for any increment $\Delta \boldsymbol{h}_{n}$, the Fréchet differential has to be positive:

$$
\delta f\left(\boldsymbol{h}_{n}, \Lambda\right)>0 .
$$

Hence, a necessary and sufficient condition which the minimum must satisfy is obtained:

$$
2 \Delta \boldsymbol{h}_{n}+\boldsymbol{X} \Lambda=0 .
$$

Taking into account (35), the previous expression can be rewritten as

$$
\boldsymbol{h}_{n}=\boldsymbol{P}_{1} \boldsymbol{h}_{n-1}-\frac{1}{2} \boldsymbol{X} \Lambda .
$$

Equations (41) and (31) make up the system of $N+K$ linear equations with $N+K$ unknowns. These unknowns are the $N$ entries of the vector $\boldsymbol{h}_{n}$ (the $n$-th line of the matrix $\tilde{\boldsymbol{H}}$ ) and $K$ Lagrange multipliers. Equations (41) and (31) conform to a system of linear equations:

$$
\left[\begin{array}{cc}
\boldsymbol{I} & \frac{1}{2} \boldsymbol{X} \\
\boldsymbol{X}^{T} & \mathbf{0}
\end{array}\right]\left[\begin{array}{c}
\boldsymbol{h}_{n} \\
\Lambda
\end{array}\right]=\left[\begin{array}{c}
\boldsymbol{P}_{\mathbf{1}} \boldsymbol{h}_{\boldsymbol{n}-\mathbf{1}} \\
\boldsymbol{y}_{n}
\end{array}\right]
$$

where $\boldsymbol{I}$ is an $N \times N$ identity matrix and $\mathbf{0}$ is a $K \times K$ zero matrix. The determinant of the system of equations (42) is given by (Meyer, 2000)

$$
\begin{aligned}
& \operatorname{det}\left[\begin{array}{cc}
\boldsymbol{I} & \frac{1}{2} \boldsymbol{X} \\
\boldsymbol{X}^{T} & \mathbf{0}
\end{array}\right] \\
& =\operatorname{det} \boldsymbol{I} \operatorname{det}\left(\mathbf{0}-\boldsymbol{X}^{T} \boldsymbol{I}^{-1} \frac{1}{2} \boldsymbol{X}\right) \\
& =\operatorname{det}\left(\frac{1}{2} \boldsymbol{X}^{T} \boldsymbol{X}\right)
\end{aligned}
$$

where $\left(\mathbf{0}-\boldsymbol{X}^{T} \boldsymbol{I}^{-1} \frac{1}{2} \boldsymbol{X}\right)$ is the so-called Schur complement. The condition for the existence of a unique solution of (42) is

$$
\operatorname{det}\left(\boldsymbol{X}^{T} \boldsymbol{X}\right) \neq 0
$$

This condition implies

$$
\operatorname{rank} \boldsymbol{X}=K, \quad\left(\boldsymbol{X} \in \mathbb{R}^{N \times K}, K \leq N\right) .
$$

In other words, linear independence of stimulus signals in the matrix $\boldsymbol{X}$ is required. If the condition (44) is fulfilled, the optimization problem has a unique solution, being the global minimum.

The linear independence condition (44) is justified from the point of view of signal selection for identification. Thus, the algorithm is protected against requirements which are contradictory to the linearity of a CPO. If the condition (44) has to be fulfilled, it is not possible to include signals which are proportional to each other in the set of input signals but with related response signals of different shape. Because of the time-varying nature of the $\mathrm{CPO}$, it is possible to obtain different response signals for the same stimuli but shifted in time. The condition (44) does not preclude this situation.

Substituting (41) to (31) and after some manipulations, the following expression results:

$$
\frac{1}{2} \Lambda=\left(\boldsymbol{X}^{T} \boldsymbol{X}\right)^{-1} \boldsymbol{X}^{T} \boldsymbol{P}_{1} \boldsymbol{h}_{n-1}-\left(\boldsymbol{X}^{T} \boldsymbol{X}\right)^{-1} \boldsymbol{y}_{n} .
$$

Substituting (46) to (41) and after ordering, an iterative solution is obtained

$\boldsymbol{h}_{n}=\left(\mathbf{I}-\boldsymbol{X}\left(\boldsymbol{X}^{T} \boldsymbol{X}\right)^{-1} \boldsymbol{X}^{T}\right) \boldsymbol{P}_{1} \boldsymbol{h}_{n-1}+\boldsymbol{X}\left(\boldsymbol{X}^{T} \boldsymbol{X}\right)^{-1} \boldsymbol{y}_{n}$

The identification of the CPO consists of an iteration scheme determining consecutive rows of the matrix $\tilde{\boldsymbol{H}}$ 
with reference to the previous rows, taking into account the optimality criterion and the periodicity of the identified system. Each row of the matrix $\tilde{\boldsymbol{H}}$ obtained from the iteration fulfills (31). The iterations should be executed until an $N$-periodic steady state is attained. It other words, after $N$ iterations, nearly the same result is obtained:

$$
\boldsymbol{h}_{n+N}=\boldsymbol{h}_{n}+\boldsymbol{\epsilon},
$$

where $\epsilon$ is a vector of satisfactorily small deviation entries.

The iteration scheme given in 477 has the form of a standard discrete state space equation:

$$
\boldsymbol{v}(n+1)=\boldsymbol{A} \boldsymbol{v}(n)+\boldsymbol{B} \boldsymbol{u}(n),
$$

where

$$
\begin{aligned}
& \boldsymbol{A}=\left(\mathbf{I}-\boldsymbol{X}\left(\boldsymbol{X}^{T} \boldsymbol{X}\right)^{-1} \boldsymbol{X}^{T}\right) \boldsymbol{P}_{\mathbf{1}}, \\
& \boldsymbol{B}=\boldsymbol{X}\left(\boldsymbol{X}^{T} \boldsymbol{X}\right)^{-1}
\end{aligned}
$$

The following two theorems will be useful to prove the convergence of the identification iteration scheme (47).

Theorem 1. If the condition (44) is fulfilled, all eigenvalues of the matrix $\boldsymbol{A} \in \mathbb{R}^{N \times N}$ defined by (50) lie on or inside the unit circle. In other words,

$$
\left|\lambda_{i}\right| \leq 1, \quad \forall 1 \leq i \leq N
$$

Proof. The following criterion, which is similar to the Lyapunov stability test for discrete time systems, is used. If there exists a positive definite matrix $\boldsymbol{P} \in \mathbb{R}^{N \times N}$ and a negative semi-definite matrix $\boldsymbol{Q} \in \mathbb{R}^{N \times N}$ such that

$$
\boldsymbol{A}^{T} \boldsymbol{P} \boldsymbol{A}-\boldsymbol{P}=\boldsymbol{Q},
$$

then all the eigenvalues of $\boldsymbol{A}$ lie on or inside the unit circle. In other words,

$$
\left|\lambda_{i}\right| \leq 1, \quad i=1,2, \ldots, N
$$

Proof of the criterion. For each eigenvalue $\lambda_{i}$ and for an appropriate eigenvector $\boldsymbol{v}_{i}$ of $\boldsymbol{A}$,

$$
\boldsymbol{A} \boldsymbol{v}_{i}=\lambda_{i} \boldsymbol{v}_{i} \text { and } \boldsymbol{v}_{i}^{*} \boldsymbol{A}^{*}=\lambda_{i}^{*} \boldsymbol{v}_{i}^{*} .
$$

Premultiplying (53) by $\boldsymbol{v}_{i}^{*}$ and postmultiplying it by $\boldsymbol{v}_{i}$, taking into account that for any matrix with real-valued entries $\boldsymbol{A}^{*}=\boldsymbol{A}^{T}$, and making use of (55), the following expression is obtained:

$$
\begin{aligned}
& \boldsymbol{v}_{i}^{*} \boldsymbol{A}^{*} \boldsymbol{P} \boldsymbol{A} \boldsymbol{v}_{i}-\boldsymbol{v}_{i}^{*} \boldsymbol{P} \boldsymbol{v}_{i} \\
& \quad=\left(\left|\lambda_{i}\right|^{2}-1\right) \boldsymbol{v}_{i}^{*} \boldsymbol{P} \boldsymbol{v}_{i}=\boldsymbol{v}_{i}^{*} \boldsymbol{Q} \boldsymbol{v}_{i} .
\end{aligned}
$$

If $\boldsymbol{P}$ is a positive definite matrix and $\boldsymbol{Q}$ is a negative semi-definite matrix, then it results from (56) that

$$
\left|\lambda_{i}\right|^{2}-1 \leq 0
$$

which leads to (54).

To prove Theorem 1 let us assume that the matrix $\boldsymbol{P}$ is the identity matrix (which is, of course, positive definite):

$$
P=\mathbf{I} \text {. }
$$

Substituting (50) and 58) into (53), and taking into account

$$
\boldsymbol{P}_{1}^{T} \boldsymbol{P}_{1}=\mathbf{I},
$$

after some calculations it is obtained that

$$
\boldsymbol{P}_{1}^{T} \boldsymbol{X}\left(\boldsymbol{X}^{T} \boldsymbol{X}\right)^{-1} \boldsymbol{X}^{T} \boldsymbol{P}_{1}=-\boldsymbol{Q} .
$$

The matrix on the left-hand side of (60) is an idempotent matrix, and therefore its eigenvalues are equal to 0 or/and 1 , hence it is a positive semi-definite matrix. This means that $Q$ is a negative semi-definite matrix. The assumptions of the criterion given previously are fulfilled, and therefore Theorem 1 has been proved.

Theorem 2. The matrix $\boldsymbol{A}$ defined by (51) has a pair of eigenvalues on the unit circle of the form

$$
\lambda_{k}=w^{-k}, \quad \lambda_{N-k}=w^{k},
$$

where $w=\exp (j 2 \pi / N)$, if and only if none of stimulus signals being the columns of the matrix $\boldsymbol{X}$ includes the $k$-th harmonic.

Proof. The necessity is proved by determining the eigenvalues of $\boldsymbol{A}$. To this end, the Fourier matrix $\boldsymbol{F}$ given in (19) is used in a similarity transformation:

$$
\begin{aligned}
\boldsymbol{A}^{\prime} & =\boldsymbol{F}^{-1} \boldsymbol{A} \boldsymbol{F}=\boldsymbol{F}^{-1}\left(\mathbf{I}-\boldsymbol{X}\left(\boldsymbol{X}^{T} \boldsymbol{X}\right)^{-1} \boldsymbol{X}^{T}\right) \boldsymbol{P}_{1} \boldsymbol{F} \\
& =\boldsymbol{F}^{-1} \boldsymbol{P}_{1} \boldsymbol{F}-\boldsymbol{F}^{-1} \boldsymbol{X}\left(\boldsymbol{X}^{T} \boldsymbol{X}\right)^{-1} \boldsymbol{X}^{T} \boldsymbol{F} \boldsymbol{F}^{-1} \boldsymbol{P}_{1} \boldsymbol{F}
\end{aligned}
$$

The result of the matrix product $\boldsymbol{F}^{-1} \boldsymbol{X}$ is a matrix whose columns contain signals in the form of a Fourier series. If none of the signals includes the $k$-th harmonic, then the $k$-th and $(N-k)$-th rows of the matrix contain zeros only. A similar property is found in the product

$$
\boldsymbol{X}^{T} \boldsymbol{F}=\boldsymbol{X}^{*} N\left(\boldsymbol{F}^{-1}\right)^{*}=N\left(\boldsymbol{F}^{-1} \boldsymbol{X}\right)^{*},
$$

which is the conjugate transpose of $\boldsymbol{F}^{-1} \boldsymbol{X}$ multiplied by the integer quantity $N$. Its $k$-th and $(N-k)$-th columns contain zeros, too. For these reasons, all the entries of the $k$-th and $(N-k)$-th rows and $k$-th and $(N-k)$-th columns of the matrix product $\boldsymbol{F}^{-1} \boldsymbol{X}\left(\boldsymbol{X}^{T} \boldsymbol{X}\right)^{-1} \boldsymbol{X}^{T} \boldsymbol{F} \boldsymbol{F}^{-1} \boldsymbol{P}_{1} \boldsymbol{F}$ 
are equal to zero. In 62 , the product $\boldsymbol{F}^{-1} \boldsymbol{P}_{1} \boldsymbol{F}$ is a diagonal matrix which is given by

$$
\boldsymbol{F}^{-1} \boldsymbol{P}_{1} \boldsymbol{F}=\left[\begin{array}{ccccc}
w^{0} & 0 & 0 & \cdots & 0 \\
0 & w^{-1} & 0 & \cdots & 0 \\
0 & 0 & w^{-2} & \cdots & 0 \\
\cdots & \cdots & \cdots & & \cdots \\
0 & 0 & 0 & \cdots & w^{N-1}
\end{array}\right]
$$

Thus, the matrix $\boldsymbol{A}^{\prime}$ given in (62) includes two rows and two columns with entries equal to zero except for diagonal entries derived from the matrix $\boldsymbol{F}^{-1} \boldsymbol{P}_{1} \boldsymbol{F}$, i.e.,

$$
\left[\boldsymbol{A}^{\prime}\right]_{k, k}=w^{-k}, \quad\left[\boldsymbol{A}^{\prime}\right]_{N-k, N-k}=w^{k}
$$

The characteristic polynomial of the matrix $\boldsymbol{A}$ can be determined as follows:

$$
\begin{aligned}
& \varphi(\lambda) \\
& =\operatorname{det}(\lambda \boldsymbol{I}-\boldsymbol{A})=\operatorname{det}\left(\lambda \boldsymbol{I}-\boldsymbol{A}^{\prime}\right) \\
& =\operatorname{det}\left(\lambda \boldsymbol{I}-\boldsymbol{F}^{-1} \boldsymbol{P}_{1} \boldsymbol{F}+\boldsymbol{F}^{-1} \boldsymbol{X}\left(\boldsymbol{X}^{T} \boldsymbol{X}\right)^{-1} \boldsymbol{X}^{T} \boldsymbol{F} \boldsymbol{F}^{-1} \boldsymbol{P}_{1} \boldsymbol{F}\right) \\
& =\operatorname{det}\left[\begin{array}{ccccc} 
& 0 & & 0 & \\
\boldsymbol{I} \lambda-\boldsymbol{A}_{11}^{\prime} & \vdots & \boldsymbol{A}^{\prime}{ }_{12} & \vdots & \boldsymbol{A}^{\prime}{ }_{13} \\
& 0 & & 0 & \\
0 \cdots 0 & \lambda-w^{-k} & 0 \cdots 0 & 0 & 0 \cdots 0 \\
& 0 & & 0 & \\
\boldsymbol{A}_{21}^{\prime} & \vdots & \boldsymbol{I} \lambda-\boldsymbol{A}_{22}^{\prime} & \vdots & \boldsymbol{A}^{\prime}{ }_{23} \\
& 0 & & 0 & \\
0 \cdots 0 & 0 & 0 \cdots 0 & \lambda-w^{k} & 0 \cdots 0 \\
& 0 & & 0 & \\
\boldsymbol{A}^{\prime}{ }_{31} & \vdots & \boldsymbol{A}_{32}^{\prime} & \vdots & \boldsymbol{I} \lambda-\boldsymbol{A}^{\prime} \\
& 0 & & 0 &
\end{array}\right] \\
& =\left(\lambda-w^{-k}\right)\left(\lambda-w^{k}\right) \\
& \cdot \operatorname{det}\left[\begin{array}{ccc}
\boldsymbol{I} \lambda-\boldsymbol{A}^{\prime}{ }_{11} & \boldsymbol{A}^{\prime}{ }_{12} & \boldsymbol{A}_{13}^{\prime} \\
\boldsymbol{A}^{\prime}{ }_{21} & \boldsymbol{I} \lambda-\boldsymbol{A}_{22}^{\prime} & \boldsymbol{A}_{23}^{\prime} \\
\boldsymbol{A}^{\prime}{ }_{31} & \boldsymbol{A}^{\prime}{ }_{32} & \boldsymbol{I} \lambda-\boldsymbol{A}^{\prime}{ }_{33}
\end{array}\right] \text {, }
\end{aligned}
$$

where $\boldsymbol{A}^{\prime}{ }_{\text {rs }}$ are sub-matrices of the matrix $\boldsymbol{A}^{\prime}$, and $\boldsymbol{I}$ means an identity matrix of a proper order. The result obtained in 66 proves the necessity in Theorem 2

The sufficiency is shown by means of an indirect proof. Assume that the matrix $\boldsymbol{X}$ includes only one signal which is a sine of the $k$-th harmonic frequency, i.e.,

$$
\boldsymbol{X}=[x(0) x(1) \cdots x(N-1)]^{T}, x(n)=\sin \left(k \frac{2 \pi}{N} n\right) .
$$

In this case,

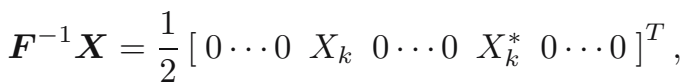

$$
\begin{aligned}
\boldsymbol{X}^{T} \boldsymbol{F} & =N\left(\boldsymbol{F}^{-1} \boldsymbol{X}\right)^{*} \\
& =\frac{N}{2}\left[\begin{array}{llllllll}
0 & \cdots 0 & X_{k}^{*} & 0 & \cdots & X_{k} & 0 & \cdots
\end{array}\right],
\end{aligned}
$$

$$
\begin{aligned}
& \boldsymbol{X}^{T} \boldsymbol{X} \\
& =[x(0) x(1) \ldots x(N-1)][x(0) x(1) \ldots x(N-1)]^{T} \\
& =N X_{k \text { rms }}^{2}=\frac{N}{2}\left|X_{k}\right|^{2},
\end{aligned}
$$

where $X_{k}$ is a complex phasor of the $k$-th harmonic, $X_{k \mathrm{rms}}$ is a root-mean-square value of the $k$-th harmonic. Thus the matrix $\boldsymbol{A}^{\prime}$ defined in (62) takes the form of the matrix product $\boldsymbol{F}^{-1} \boldsymbol{P}_{\mathbf{1}} \boldsymbol{F}$ (64) with except for the entries:

$$
\begin{aligned}
{\left[\boldsymbol{A}^{\prime}\right]_{k, k} } & =\frac{w^{-k}}{2} \\
{\left[\boldsymbol{A}^{\prime}\right]_{k, N-k} } & =-\frac{X_{k}^{2}}{2\left|X_{k}\right|^{2}} w^{-(N-k),} \\
{\left[\boldsymbol{A}^{\prime}\right]_{k-N, k} } & =-\frac{\left(X_{k}^{*}\right)^{2}}{2\left|X_{k}\right|^{2}} w^{-k} \\
{\left[\boldsymbol{A}^{\prime}\right]_{N-k, N-k} } & =\frac{w^{-(N-k)}}{2} .
\end{aligned}
$$

The characteristic polynomial of the matrix $\boldsymbol{A}$ can be determined as follows:

$$
\begin{aligned}
& \varphi(\lambda) \\
& =\operatorname{det}(\lambda \boldsymbol{I}-\boldsymbol{A})=\operatorname{det}\left(\lambda \boldsymbol{I}-\boldsymbol{A}^{\prime}\right) \\
& =\prod_{n=0}^{k-1}\left(\lambda-w^{-n}\right) \cdot \prod_{n=k+1}^{N-k-1}\left(\lambda-w^{-n}\right) \cdot \prod_{n=N-k+1}^{N-1}\left(\lambda-w^{-n}\right) \\
& \quad \cdot \operatorname{det}\left[\begin{array}{c}
\frac{w^{-k}}{2}-\lambda \quad-\frac{X_{k}^{2}}{2\left|X_{k}\right|^{2}} w^{-(N-k)} \\
-\frac{\left(X_{k}^{*}\right)^{2}}{2\left|X_{k}\right|^{2}} w^{-k} \\
\frac{w^{-(N-k)}}{2}-\lambda
\end{array}\right] \\
& =\prod_{n=0}^{N-k-1}\left(\lambda-w^{-n}\right) \cdot \prod_{n=k+1}^{N-1}\left(\lambda-w^{-n}\right) \cdot \prod_{n=N-k+1}^{N}\left(\lambda-w^{-n}\right) \\
& \cdot \lambda\left(\lambda-\cos \left(k \frac{2 \pi}{N}\right)\right) .
\end{aligned}
$$

The obtained form of the characteristic polynomial (72) indicates that, under the assumption of the indirect proof, the matrix $\boldsymbol{A}$ does not have any eigenvalues of the form given in 61]. This is contradictory to the assumption of Theorem 2 and proves the sufficiency in this theorem.

Some eigenvalues of the matrix $\boldsymbol{A}$ given in $(50)$ may lie on the unit circle, and the remaining eigenvalues are located inside it. This means that the iteration scheme presented in 47) does not have the property of asymptotic convergence. The solution of the homogenous equation

$$
\boldsymbol{v}(n+1)=\boldsymbol{A} \boldsymbol{v}(n)
$$


can have a non-decaying periodic component. In order to avoid it, the initial condition of the iterations 47 has to be a zero vector:

$$
\boldsymbol{h}_{0}=\left[\begin{array}{llll}
0 & 0 & \cdots & 0
\end{array}\right]^{T} .
$$

The convergence of the iteration scheme given in 47) to perform the identification of the $\mathrm{CPO}$ associated with (11) depends on the input term present in (49):

$$
\boldsymbol{B} \boldsymbol{u}(n)=\boldsymbol{X}\left(\boldsymbol{X}^{T} \boldsymbol{X}\right)^{-1} \boldsymbol{y}_{n}
$$

If some eigenvalues of the matrix $\boldsymbol{A}$ lie on the unit circle, the iterations (47) may lose convergence. This is possible if the input term includes the harmonic of angular frequency equal to any of these eigenvalues. The input term given in (75) can be treated as a linear combination of signals included in $\boldsymbol{X}$ with coefficients obtained from the matrix operation $\left(\boldsymbol{X}^{T} \boldsymbol{X}\right)^{-1} \boldsymbol{y}_{n}$. If none of signals being a column of the matrix $\boldsymbol{X}$ includes the $k$-th harmonic, then their linear combination does not include this harmonic as well. Taking into account Theorem 2 , the presence of eigenvalues on the unit circle of the form given in (61) means the lack of the $k$-th harmonic in the input component given in (75). Thus, the solution of the equation given in 49) converges to a periodic signal such that the identification scheme given in (47) is convergent. A periodic solution of the identification iterations implies periodic variability of parameters of the system described by the determined CPO. The numerical experiments carried out so far confirm the convergence properties of the algorithm.

\section{Numerical experiments}

Some numerical experiments were carried out to verify the validity and properties of the identification iteration scheme. An original CPO of a simple one-port network was determined by means of numerical simulation methods. The diagram of the one-port network and the functions defining the changes of the resistance, inductance and capacity are presented in Figs. 2 and 3 . The resistance changes function is a double frequency sine with a direct component, the inductance changes function is a triple frequency sine with a direct component, and the capacity changes function is a sine of a frequency four times higher the basic input frequency with a direct component. The graphs of the impedance CPO $\tilde{Z}(n, m)$ and of the module of the SCPO $\left|\overline{\tilde{\boldsymbol{H}}}\left(k_{U}, k_{I}\right)\right|$ are presented in Figs. 4 and 5

Next, the set of current signals used as stimuli was generated. The response voltage signals were obtained by applying the original CPO on the stimuli. Using the proposed iteration scheme, the identified impedance CPO was determined, and using 2D DFT, the identified SCPO

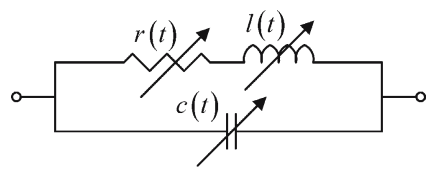

Fig. 2. Diagram of the LPTV one-port network.

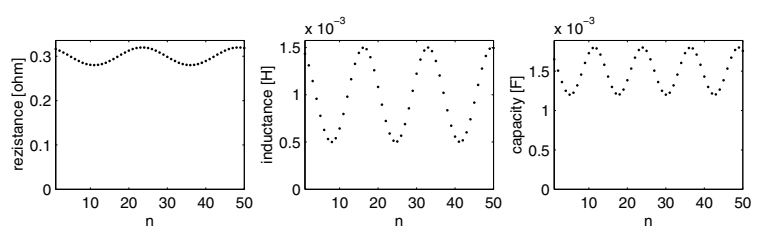

Fig. 3. Periodic functions defining parameter changes of the elements present in the one-port network of Fig. 2

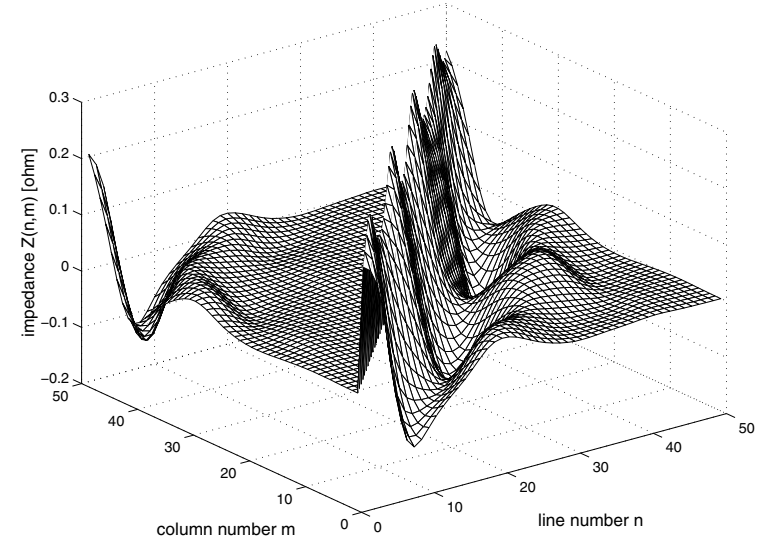

Fig. 4. Original impedance CPO of the LPTV one-port network of Fig. 2

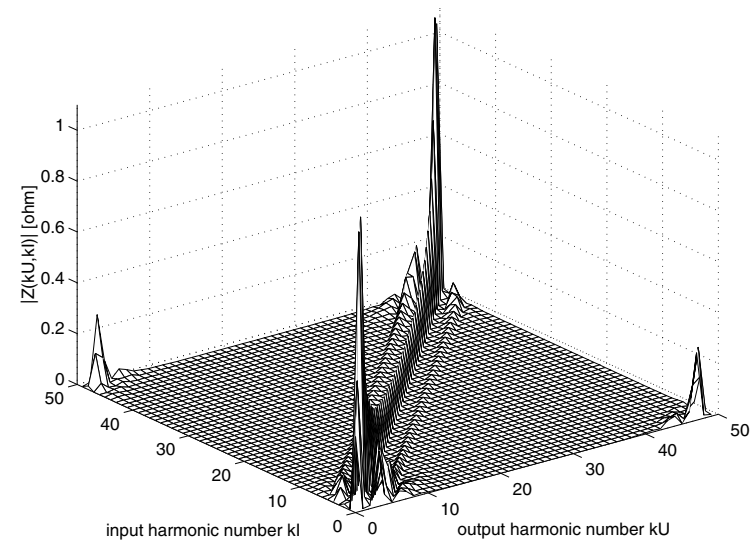

Fig. 5. Module of the original SPCO of the LPTV one-port network.

was calculated. Then, the identification iteration scheme effectiveness test was carried out. The response voltage 


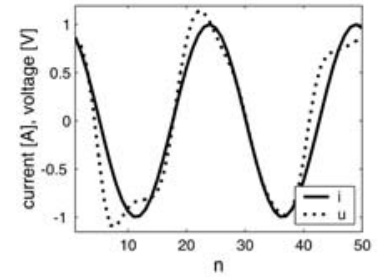

(a)

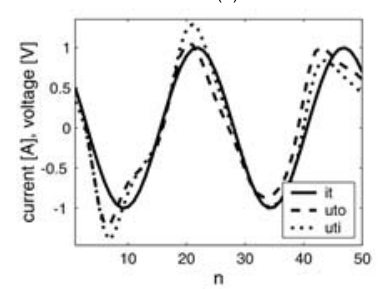

(c)

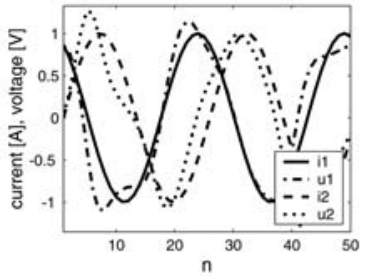

(b)

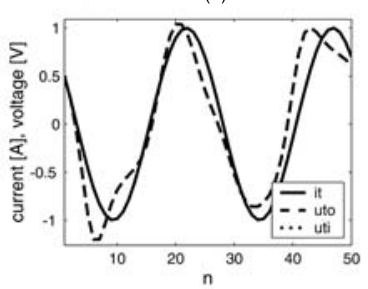

(d)
Fig. 6. Results of the test performed on the identified CPO by means of sine stimulus: (a) one sine stimulus current signal and the response voltage signal, (b) two sine current stimulus signals with different phases and response voltage signals, (c) and (d) comparison of the response voltage signals obtained by applying the original operator ("uto") and the identified operator ("uti") on the test sine stimulus signal ("it").

signals obtained by applying the original operator (which is marked in Figs. 6 and 12, 15 with "uto") and the identified operator ("uti") on some test stimulus signals ("it") were compared. The identification based on sine stimulus signals was carried out. The CPO obtained by means of one sine current (symbol "i") and its associated voltage response (symbol "u") presented in Fig. 6a) yields a wrong test response voltage signal to the sine stimulus shifted in time presented in Fig. 6(c). Making use of two sine current stimuli with different phases presented as "i1" and "i2" in Fig. 6. (b) yields the identified CPO, which operates on the test stimulus in the same way as the original one, see Fig. 6(d). The graphs of the identified CPO and of the SCPO module are presented in Figs. 7 and 8 The usage of two double frequency sine stimuli allowed to determine two columns of the SCPO related to the 2-nd harmonic of stimulus. One of the columns can be treated as the impedance of the LPTV one-port network for exact frequency. It allows us to determine voltage harmonics phasors, when the one-port network is supplied with sine current. The second column determined carries the same information, because it consists of conjugated entries of the first one.

The distribution of eigenvalues of the matrix $\boldsymbol{A}$ in (50) on the complex plane is presented in Fig. 9. According to Theorem 2, the presence of only one harmonic in the set of stimuli results in almost all eigenvalues lying on the unit circle.

The next example demonstrates the application of

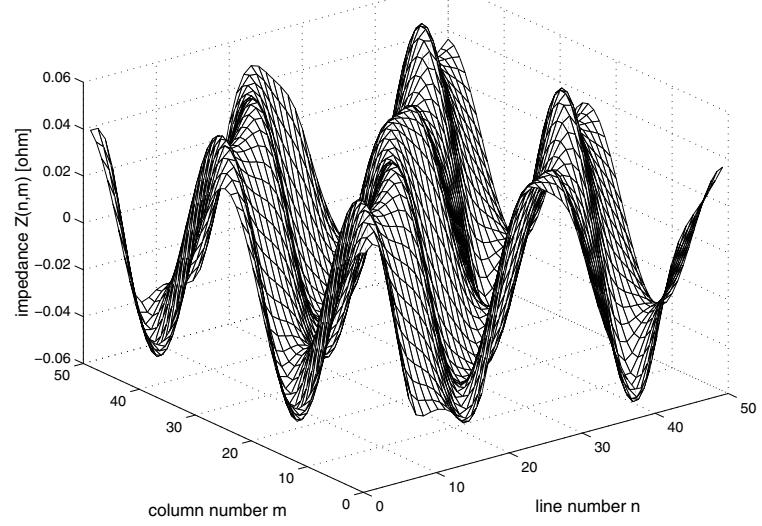

Fig. 7. CPO obtained from identification based on two sine stimulus signals.

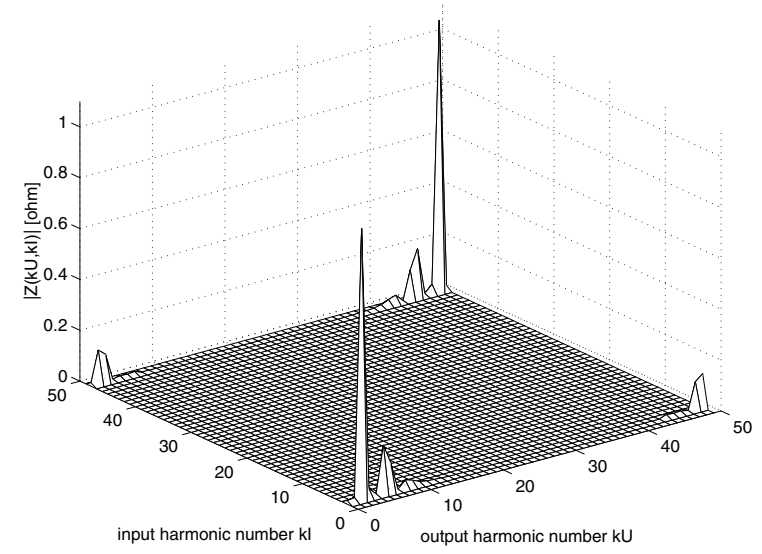

Fig. 8. Module of the SCPO obtained from identification based on two sine stimulus signals.
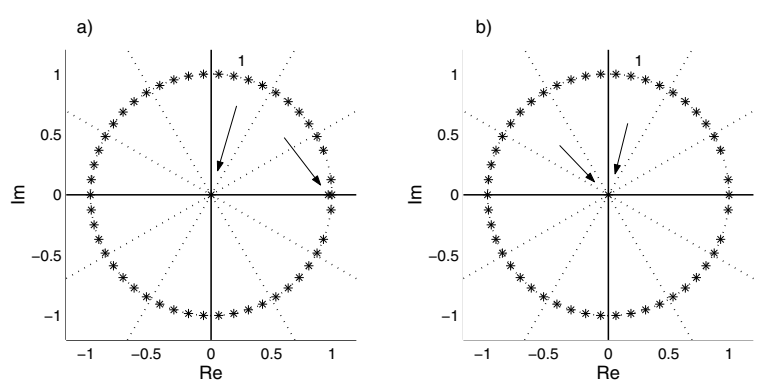

Fig. 9. Distribution of eigenvalues of the matrix $\boldsymbol{A}$ : (a) when one double frequency sine stimulus signal is used, (b) when two sine stimulus signals of the same double frequency but different phase are used. Arrows show eigenvalues of the module less than 1 .

polyharmonic stimulus signals to identify the CPO. Random signals of uniformly distributed samples in the interval $(0,1)$ were used. When one random signal, which 


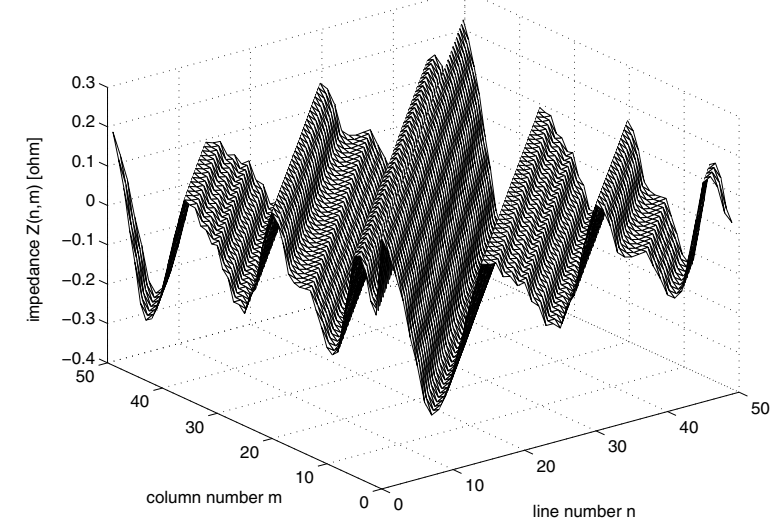

Fig. 10. CPO obtained from identification based on one random stimulus signal.

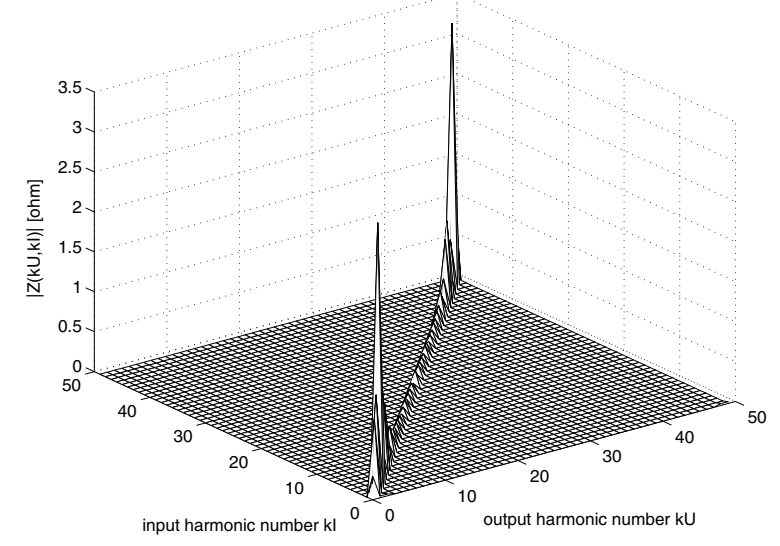

Fig. 11. Module of the SCPO obtained from identification based on one random stimulus signal.

contains all harmonics of order $0, \ldots, N / 2$ is used, then the CPO obtained from identification takes the form of a circular matrix and the SCPO takes that of a diagonal one. This is typical of an LTI system. Graphs of the obtained CPO and SCPO are presented in Figs. 10 and 11 The test results presented in Fig. 12 show that using one signal pair yields poor identification effects. Obtained from 3, 10 and 30 random stimulus signals, the CPO identification test results are presented in Figs. 13, 14 and 15, respectively.

The CPO and the SCPO obtained by means of 30 signal pairs are shown in Figs. 16 and 17. Comparing these to Figs. 4 and 5] and taking into account that rank of the $\mathrm{CPO}$ is 50 , it seems that system identification on the basis of an incomplete set of signals using the presented iteration scheme can provide correct results.
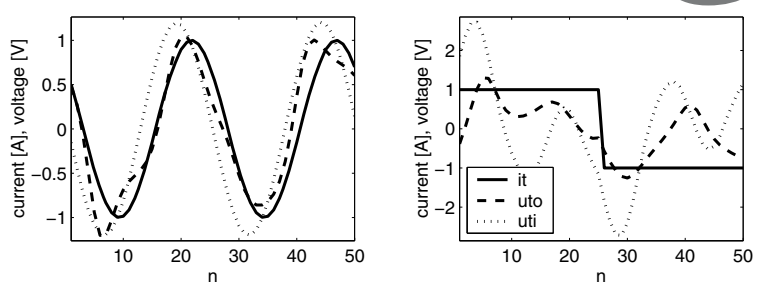

Fig. 12. Results of the test performed on the identified CPO by means of one random stimulus signal.
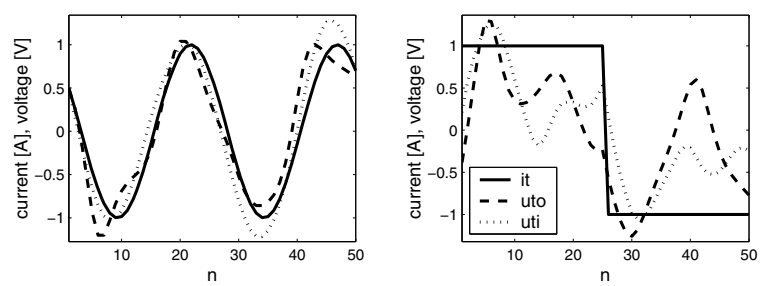

Fig. 13. Module of the SCPO obtained from identification based on three random stimulus signals.
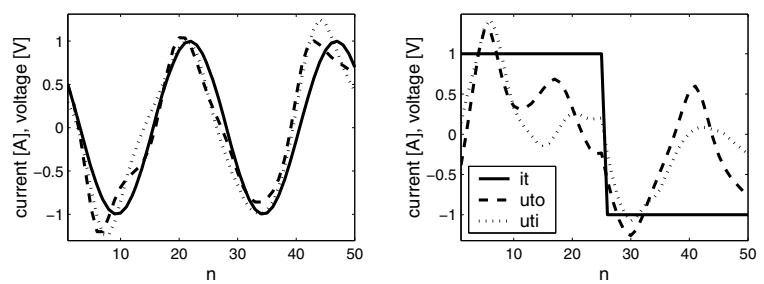

Fig. 14. Results of the test performed on the identified CPO by means of ten random stimulus signals.
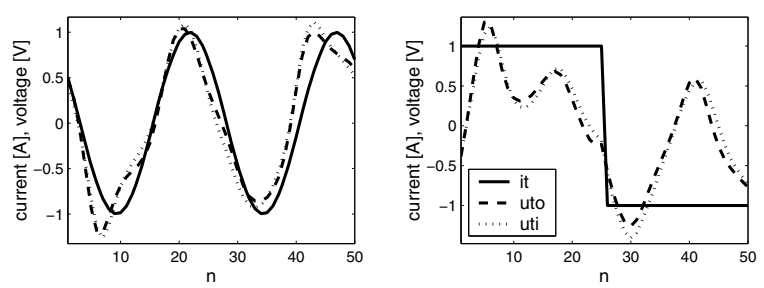

Fig. 15. Module of the SCPO obtained from identification based on 30 random stimulus signals.

\section{Conclusion}

A circular parametric operator can be used to describe the relation between terminal signals of a periodically time-varying one-port network operating in a steady-state regime. Within the domain of discrete time, such an operator takes the form of a matrix with real-valued entries. A spectral circular parametric operator may be used for quantitative assessment of input and output harmonics aliasing, which is a characteristic phenomenon in timevarying and non-linear systems. An important advantage of the representation of an LPTV system by means of the $\mathrm{SCPO}$, at least from the point of view of an electrical en- 


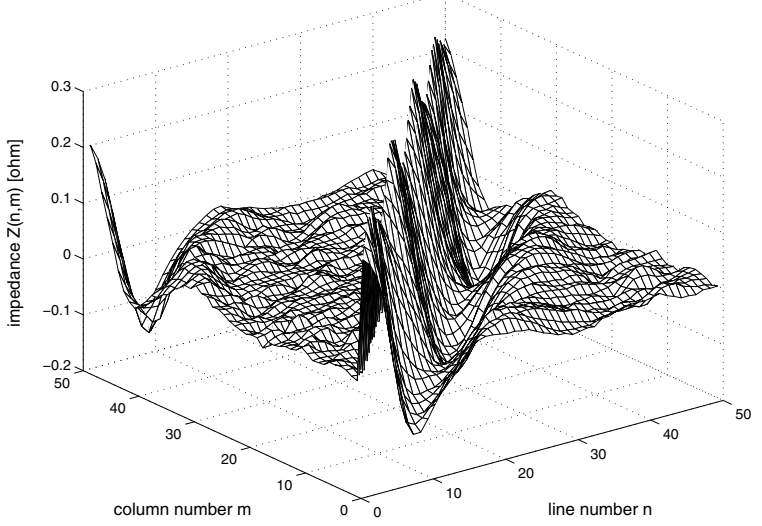

Fig. 16. CPO obtained from identification based on 30 random stimulus signals.

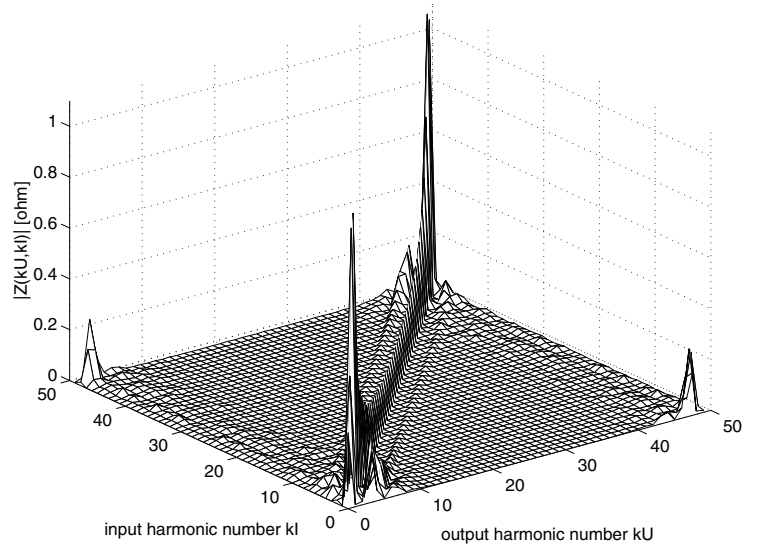

Fig. 17. Module of the SCPO obtained from identification based on 30 random stimulus signals.

gineer, is its similarity to the frequency response of an LTI system. However, differences between both representations are visible. The described iterative identification scheme can be used to determine the time-varying oneport network impedance circular parametric operator. The proposed scheme is a convenient way to determine the $\mathrm{CPO}$ and the SCPO of a given system from a set of measurements. It yields expected results, provided that the identification data uses includes the required information about the performance of the system being identified.

\section{References}

Bittanti, S. and Colaneri, P. (1999). Periodic Control. John Wiley Encyclopedia of Electrical and Electronic Engineering, Wiley, New York, NY.

Bittanti, S. and Colaneri, P. (2000). Invariant representations of discrete-time periodic systems, Automatica 36(12): 17771793.
Bru, R., Romero, S. and Sanchez, E. (2004). Structural properties of positive periodic discrete-time linear systems: Canonical forms, Applied Mathematics and Computation 153(3): 697-719.

Chen, T. and Qiu, L. (1997). Linear periodically time-varying discrete-time systems: Aliasing and LTI approximations, Systems and Control Letters 30(5): 225-235.

Doroslovacki, M., Fan, H. and Yao, L. (1998). Wavelet-based identification of linear discrete-time systems: Robustness issue, Automatica 34(12): 1637-1640.

Hu, S., Meinke, K., Chen, R. and Huajiang, O. (2007). Iterative estimators of parameters in linear models with partially variant coefficients, International Journal of Applied Mathematics and Computer Science 17(2): 179-187.

Kaczorek, T. (2001). Externally and internally positive timevarying linear systems, International Journal of Applied Mathematics and Computer Science 11(4): 957-964.

Kłosiński, R. (2005). Application of an identification algorithm for optimal control of compensation circuits, Proceedings of the International Conference on Power Electronics and Intelligent Control for Energy Conservation, PELINCEC 2005, Warsaw, Poland, pp. 1-6.

Kłosiński, R. (2006). Periodically time-varying two-terminals at a steady state, description and identification, Proceedings of the 13th IEEE International Conference on Electronics, Circuits and Systems, ICECS 2006, Nice, France, pp. 284-287.

Kłosiński， R. (2007). Periodically variable two-terminal impedance description and measuring methods, Metrology and Measurement Systems 14(2): 375-390.

Kłosiński, R. and Kozioł, M. (2007). Reconstruction of nonlinearly deformed periodic signals using inverse circular parametric operators, Proceedings of the IEEE Instrumentation and Measurement Technology Conference, IMTC 2007, Warsaw, Poland, pp. 1-6.

Liu, K. (1997). Identification of linear time-varying systems, Journal of Sound and Vibration 204(4): 487-505.

Liu, K. (1999). Extension of modal analysis to linear time-varying systems, Journal of Sound and Vibration 226(1): 149-167.

Liu, K. and Deng, L. (2005). Experimental verification of an algorithm for identification of linear time-varying systems, Journal of Sound and Vibration 279(1): 1170-1180.

Liu, M., Tse, C. K. and Wu, J. (2003). A wavelet approach to fast approximation of steady-state waveforms of power electronics circuits, International Journal of Circuit Theory and Applications 31(6): 591-610.

Mehr, A. S. and Chen, T. (2002). Representations of linear periodically time-varying and multirate systems, IEEE Transactions on Signal Processing 50(9): 2221-2229.

Meyer, C. D. (2000). Matrix Analysis and Applied Linear Algebra, SIAM, Philadelphia, PA.

Meyer, R. A. and Burrus, C. S. (1975). A unified analysis of multirate and periodically time-varying digital filters, IEEE Transactions on Circuits and Systems 22(3): 162-168. 
Mikołajuk, K. and Staroszczyk, Z. (2003). Time-frequency approach to analysis of time varying dynamic systems, Przeglad Elektrotechniczny 79(10): 764-767.

Mikołajuk, K. and Staroszczyk, Z. (2004). Periodical variability in power systems: Small-signal models, L'Energia Electtrica 81(5-6): 97-102.

Shenoy, R. G., Burnside, D. and Parks, T. (1994). Linear periodic systems and multirate filter design, IEEE Transactions on Signal Processing 42(9): 2242-2256.

Siwczyński, M. (1987). Analysis of nonlinear systems with concentrated and distributed parameters by a new numerical operator method in time and frequency domain, Proceedings of Internationales Wissenschaftliches Kolloquium 1987, Ilmenau, Germany, pp. 759-762.

Siwczyński, M. (1995). Optimization Methods in Power Theory of Electrical Networks, Monograph No. 183, Cracow University of Technology, Cracow, (in Polish).

Siwczyński, M. and Kłosiński, R. (1997a). Current and voltage wave-form optimization with non-linear deformations for real voltage sources, COMPEL 16(2): 71-83.

Siwczyński, M. and Kłosiński, R. (1997b). Synthesis of linear inertialless periodically time varying circuits for optimal compensation of non-linear distortions, Proceedings of the 9th International Symposium on Theoretical Electrical Engineering, ISTET'97, Palermo, Italy, pp. 560-563.

Siwczyński, M., Pasko, M. and Kłosiński, R. (1993). Current minimization of the non-ideal voltage source with periodically time-varying parameters by means of an active compensator, Applied Mathematics and Computer Science 3(2): 329-340.
Staroszczyk, Z. (2002). Power system nonstationarity and accurate power system identification procedures, Proceedings of the International Conference on Harmonics and Quality of Power, Rio de Janeiro, Brasil, pp. 1-8.

Staroszczyk, Z. and Mikołajuk, K. (2004). Periodically time variance power systems impedance-Description and identification, Przeglad Elektrotechniczny 80(6): 521-526.

Tam, K. C., Wong, S. C. and Tse, C. K. (2006). A wavelet-based piecewise approach for steady-state analysis of power electronics circuits, International Journal of Circuit Theory and Applications 34(5): 559-582.

Verhagen, M. and Yu, X. (1995). A class of subspace model identification algorithms to identify periodically and arbitrarily time-varying systems, Automatica 31(2): 201-216.

Zadeh, L. A. (1950). Frequency analysis of variable networks, Proceedings of the Institute of Radio Engineers 38: $291-299$.

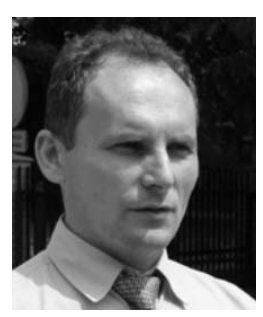

Radosław Kłosiński received the M.Sc. degree from the Technical University of Zielona Góra, Poland, in 1988, and the Ph.D. degree from the Silesian University of Technology, Gliwice, Poland, in 1994, all in electrical engineering. Since 1995, he has been an assistant professor at the University of Zielona Góra. His research interests are in periodically timevarying and non-linear systems and their applications in electrical engineering and digital signal processing. He is also interested in improving the accuracy of spectrum estimation of noncoherently sampled periodic signals.

Received: 28 August 2008

Revised: 14 April 2009 